\title{
Cylindrical space Fourier-Bessel mode solver for Maxwell's wave equation
}

\author{
Robert Claude Gauthier*, Mohammed Alzahrani \\ Dept. of Electronics, Carleton University, Ottawa, Ontario, Canada K1S 5B6 \\ Email address: \\ gauthier@doe.carleton.ca(R. C. Gauthier)
}

\section{To cite this article:}

Robert Claude Gauthier, Mohammed Alzahrani. Cylindrical Space Fourier-Bessel Mode solver for Maxwell's Wave Equation. Advances in Materials. Vol. 2, No. 3, 2013, pp. 32-35. doi: 10.11648/j.am.20130203.12

\begin{abstract}
A Fourier-Bessel basis set in cylindrical coordinates is used to cast Maxwell's wave equations into an eigenvalue problem from which the steady states of rotationally symmetric photonic structures can be determined. The rotational symmetry of the structure significantly reduces the order of the matrix making an efficient computation process that can be accommodated by desk top computers running MATLAB C. In addition the matrix can be tuned to a particular mode profile type such as monopoles, dipoles, ... enabling the user to target the desired mode features in the computations. The technique is applied to solving for the states of three different photonic structures; 12-fold quasi-crystal, silicon ring resonator and photonic crystal fiber. The particular features of a modal state are easily obtained by examining the eigenvector.
\end{abstract}

Keywords: Fourier-Bessel, Mode Solver, Photonic Crystal, Eigenmatrix, Photonic Quasi-Crystal

\section{Introduction}

Many photonic structures used in integrated optic devices display a rotational symmetry about a central reference point in a $2-\mathrm{D}$ plane. The most notable of these are the photonic crystals[1] having 2,3,4 and 6 fold rotational symmetries, photonic quasi-crystal[2] which may have any rotational order and ring resonators[3] that have infinite rotational order. When the structures are axially extended perpendicular to the plane they can be easily represented in cylindrical coordinates. A uniform extension results in the traditional 2-D photonic crystals and photonic crystal fibers[4]. When the out of plane extension contains structure such as multiple layers, cavities can be formed such as for laser resonators, ring resonators and VCSELS. The objective of this presentation is to present a numerical computation technique suitable for determining the in-plane centrally localized steady states which may contain an out of plane $z$ directed wave-vector component. The technique is based on expanding the fields, $(\vec{E}, \vec{H})$, and the inverse of the dielectric, $\Omega=1 / \varepsilon$, using a set of orthogonal basis functions generated from the product of the lowest order Bessel functions, $J_{o}$, in the radial direction and complex exponential in the angular and azimuthal directions. Due to the choice of a coordinate system that matches the in-plane rotational symmetry present, the resulting eigenvalue equation matrix order is significantly reduced and the solution process can be tuned to a particular mode type (monopoles, dipoles, quadrupoles, ... ). The next section provides the key steps in obtaining the eigenvalue expression starting from the vector wave equations. This is followed by a few numerical examples in which the steady states, $\left(k_{z}=0\right)$, of a 2-D photonic quasi-crystal and 3-D ring resonator, and the propagated fields, $\left(k_{z} \neq 0\right)$, for a microstructures photonic crystal fiber are obtained.The broad application base of the 3-D Fourier-Bessel technique presented can easily be implemented, complementing existing design and simulation software researcher tools and applied to a large number of cylindrically symmetric dielectric profiles.

\section{Eigenvalue Equation}

The wave equations are produced from Maxwell's equations for non-magnetic medium free of charges and currents:

$$
\frac{1}{\varepsilon(\vec{r})} \vec{\nabla} \times \vec{\nabla} \times \vec{E}=\left(\frac{\omega}{c}\right)^{2} \vec{E}, \quad \vec{\nabla} \times \frac{1}{\varepsilon(\vec{r})} \vec{\nabla} \times \vec{H}=\left(\frac{\omega}{c}\right)^{2} \vec{H}
$$

The eigenvalue equation is developed for $\vec{H}=H_{r} \hat{r}+$ $H_{\varphi} \hat{\varphi}+H_{z} \hat{z}$. Since both equations differ only by the placement of the relative dielectric, the electric field expression (in form) can be obtained from the magnetic field equation by setting derivative of the dielectric to 
zero.The inverse dielectric and each field component are expanded in a cylindrical coordinate equivalent of a Fourier Bessel series:

$$
\begin{aligned}
& \frac{1}{\varepsilon(\vec{r})}=\Omega=\sum_{p q n_{\Omega}} \kappa_{p q n_{\Omega}}^{\Omega} J_{o}\left(\rho_{p_{\Omega}} \frac{r}{R}\right) e^{j q_{\Omega} \varphi} e^{j G_{n_{\Omega}} z} \\
& H_{i}=\sum_{p q n_{i}} \kappa_{p q n_{i}}^{i} J_{o}\left(\rho_{p_{i}} \frac{r}{b}\right) e^{j q_{i} \varphi} e^{j\left(G_{n_{i}}+k_{z}\right) z}
\end{aligned}
$$

In these expansions, $\boldsymbol{\kappa}$ are the expansion coefficients, $\boldsymbol{p q n}$ the expansion indices, $\boldsymbol{\rho}$ the zeros of the Bessel function, $\boldsymbol{G}_{\boldsymbol{n}_{\boldsymbol{i}}}$ the expansion spatial frequencies in the $\mathrm{z}$ direction only, $\boldsymbol{k}_{\mathbf{z}}$ the z directed propagation constant and $\boldsymbol{b}$ is the radius of the dielectric structure considered.The eigenvalue equation is obtained by introducing the expansion series into (1), working through all derivatives and then forming three equations, one for each of the coordinate unit vector directions. The orthogonality of the basis functions is then made use and the resulting expressions can be written in matrix form[7]:

$$
\left[\begin{array}{ccc}
R_{r} & \varphi_{r} & Z_{r} \\
R_{\varphi} & \varphi_{\varphi} & Z_{\varphi} \\
R_{Z} & \varphi_{z} & Z_{z}
\end{array}\right]\left[\begin{array}{l}
\mathrm{R} \\
\Phi \\
Z
\end{array}\right]=\left(\frac{\omega}{c}\right)^{2} I\left[\begin{array}{l}
\mathrm{R} \\
\Phi \\
Z
\end{array}\right]
$$

The column vector $\left[\begin{array}{l}\mathrm{R} \\ \Phi \\ Z\end{array}\right]$, multiplied by the identity matrix $I$,is formed from the basis functions written in increasing index order and starting with the radial field block, $\mathrm{R}$, followed by the angular block, $\Phi$ and the azimuthal block, $Z$. These match up with the right hand field components of (1). The square matrix on the left of (3)has the radial field equation along the first row, angular field equation along the second row and azimuthal field equation along the third row. The subscript indicates the row designation. Because of the double curl process, field components on the left side of (1) are rotated and the subscripts in the matrix elements represent which coupling that takes place. The diagonal elements are the self-coupling terms and the off diagonal elements are the cross coupling terms. The expressions for the elements in the square matrix are provided in Appendix A.

\subsection{Numerical Computation Process}

The eigenvalue equation (3) can be solved for the frequencies, $\omega$, using the eig() function in MATLAB $C$. The angular dielectric decomposition index, $q_{\Omega}$, has non-zero expansion coefficients which are an integer multiple of the dielectrics rotational symmetry. Due the mixing of dielectric and field expansion series, the field index, $q_{i}$, is linked to the dielectric index $q_{\Omega}$, through $q_{i}+q_{\Omega}=$ $q_{i^{*}}$ (see Appendix A). For instance, if the dielectric has rotational symmetry of 12 in the 2-D plane, then:

$$
q_{\Omega}=\{0, \pm 12, \pm 24, \pm 36, \ldots\} .
$$

When searching for steady state solutions with pole order Q, the angular field indices are related through:

$$
q_{i}=q_{\Omega} \pm Q
$$

This relationship allows the original matrix to be segmented into individual steady state pole matrices and solved at a much reduced matrix order.

\section{Computation Examples}

\subsection{Steady States 2-D 12-Fold Quasi-Crystal}

Fig. 1 shows the 12-fold quasi-crystal plotted in the $(r, \varphi)$ plane composed of circular air holes (black) in a silicon background (white). Due to the 12-fold symmetry the expansion coefficients are restricted to the set given by (4) and since the structure is infinite in extent along the z-axis, $G_{n_{\Omega}}=\{0\}$. The field components also have $G_{n_{i}}=\{0\}$ and $q_{i}$ are related through (5). Fig. 2 shows the TM polarized eigen-wavelengths $\left(\lambda=\frac{\omega}{2 \pi c}\right)$ in the 1 to $2 \mu \mathrm{m}$ range computed using (3) for monopoles and for dipoles. The expansion indices used are $\{p, q, n\}=\{150, \pm 90,1\}$ Note that (3) returns as many eigenvalues as there are basis functions used in the expansions. Examination of the dominant field expansion coefficients in the eigenvector enables the steady state to be classified as TM or TE. When dipoles or higher mode orders are solved for the eigen-frequency solutions show up in pairs due to the $\mathrm{CW}$ and $\mathrm{CCW}$ degeneracy. Three monopole mode $H_{z}$ field profiles (intensity in grey scale) are plotted in the insert of Fig. 2.

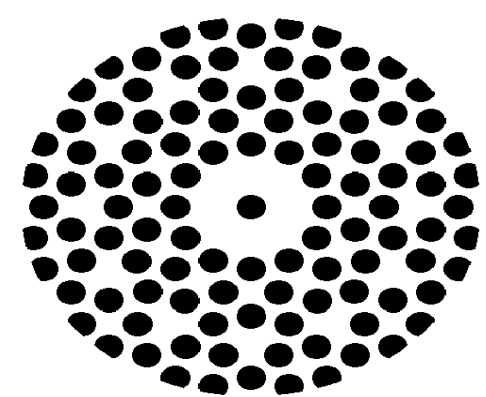

Figure 1. 12-fold quasi-crystal air hole pattern in silicon $\left(\epsilon_{r}=12.1104\right)$. radius of the structure is $2.5 \mu \mathrm{m}$.

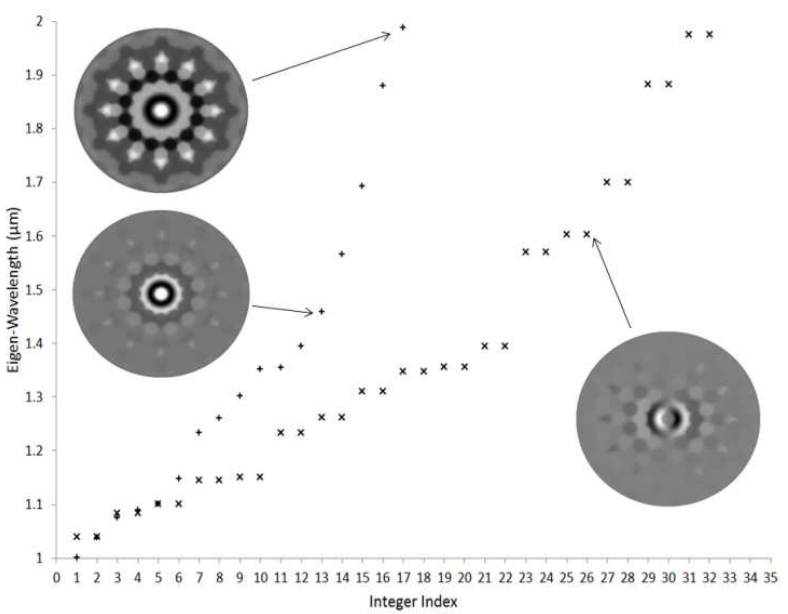

Figure 2. TM polarized states obtained from (3) in the 1 to $2 \mu \mathrm{m}$ range for monopoles (+) and dipoles (X). Two of the monopoles and a dipole field profiles are plotted, obtained using the eigenvector associated with the corresponding eigen-wavelength. 


\subsection{3-D Ring Waveguide Modes}

Fig. 3 shows the 3-D representation of the silicon ring waveguide considered. The waveguide has a 0.25 by 0.50 $\mu \mathrm{m}$ rectangular cross-section and an inner edge radius of 2 $\mu \mathrm{m}$. The expansion indices used $\operatorname{are}\{p, q, n\}=\{50,1, \pm 20\}$. The eigenmatrix (3) was solved for a mode with rotational symmetry of 20 and 24 . Fig. 4 shows three highly confined modes, wavelength $1.311 \mu \mathrm{m}$ (radial dipole, rotational order 24), wavelength $1.390 \mu \mathrm{m}$ (radial dipole, rotational order 20) and wavelength $1.444 \mu \mathrm{m}$ (radial monopole, rotational order 20). These profiles are reconstructed from their eigenvectors and plotted in the $(r, \phi, 0)$ plane. These modes are typical of modes observed in ring waveguides using other numerical and experimental techniques.

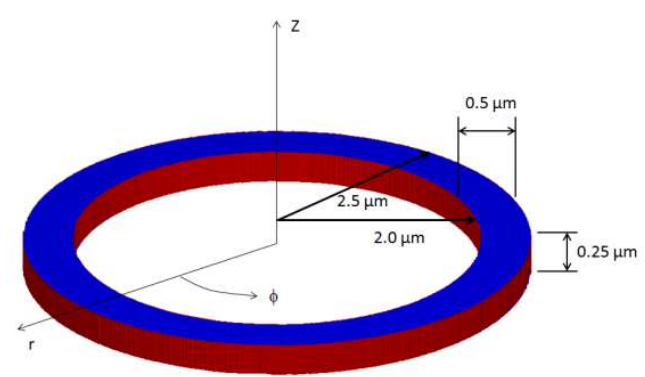

Figure 3. 3-D representation of the ring resonator examined using the Fourier-Bessel mode solving technique.
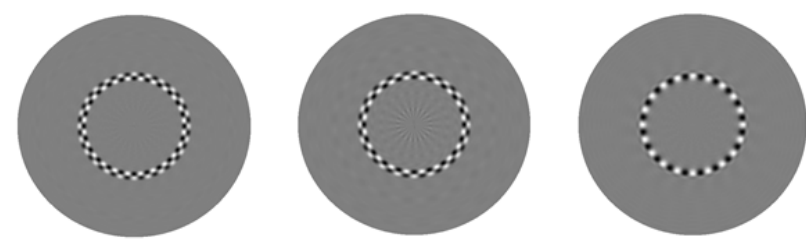

Figure 4. Three highly confined modes of the ring resonator determined for mode rotational order $24(\lambda=1.311 \mu \mathrm{m}$, left $)$ and rotational order 20 $(\lambda=1.390 \mu \mathrm{m}$, center, $\lambda=1.444 \mu \mathrm{m}$ right $)$.

\subsection{Waveguide Modes of 16-fold Perfect Periodic Photonic Quasi-Crystal Fiber}

The $(r, \varphi)$ plane dielectric profile of the photonic crystal fiber having equal periodicity in the angular and radial direction is shown in Fig. 5. The term perfect periodic photonic quasi-crystal relates to the dual equal periodicity. In the $(r, \varphi)$ polar space, over the extent of the dielectric a lattice and basis can be defined in a similar way to translational periodic crystals. The central ring of holes is masked out with silicon as these holes would be poorly defined when the structure is discretized.

The propagated modes of the fiber can be obtained by solving (3) with the $k_{z}$ propagation constant as parameter and for the particular mode profile of interest.Fig. 6 shows the plot of mode wavelength versus z-axis propagation constant for the field profiles dominated by the $H_{z}$ field component and of the monopole class. Centrally localized states can be determined by reconstructing the field using the eigenvectors. Two of these are shown in the insert and for $k_{z}=0$ the mode lines start at $1.590 \mu \mathrm{m}$ and $1.012 \mu \mathrm{m}$.
The plot also shows a mode with rotational order 16 also solved in the monopole class. This plot and the reconstruction of the field profiles indicate that the Fourier-Bessel eigen-matrix approach can be used to extract information on guided modes for highly micro structured fibers. The larger than normal number of mode lines displayed results from the technique solving for all modes of a particular mode rotational order. These include the usual centrally localized states and the surface states and the boundary states. Examination of the eigenvector efficiently sorts the modes into either category.

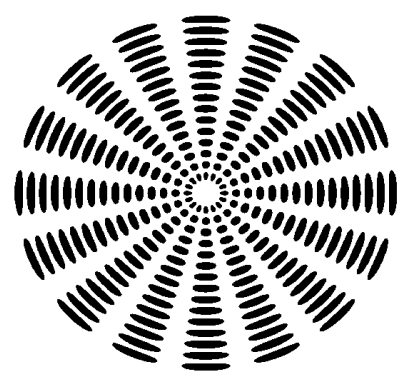

Figure 5. 16-fold perfect-periodic photonic quasi-crystal. Structure has 16-fold rotational symmetry and periodicity of 16 in the radial direction up to the $2.5 \mu \mathrm{m}$ radial boundary.

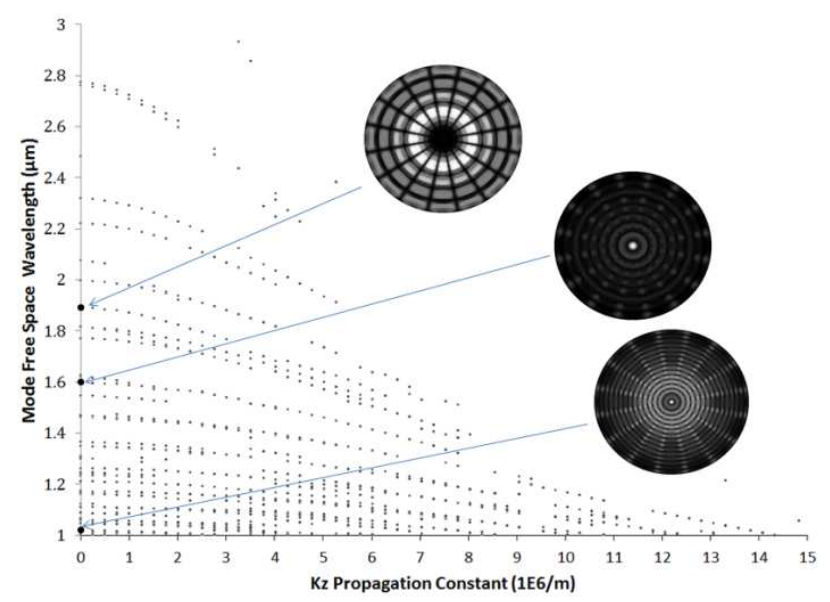

Figure 6.Plot of mode wavelength obtained from (3) verse z-axis propagation constant for the monopole solutions. Mode lines are observed and three of the centrally localized field profiles are plotted from the eigenvectors.

\section{Conclusion}

A Fourier-Bessel mode solving technique was developed in cylindrical coordinate space where Maxwell's wave equation is configured into an eigenvalue problem. The rotational symmetry of the dielectric profile about the cylinder axis enables the eigenmatrix to be significantly reduced in order making the computation of the steady states a computational efficient process. In addition the eigenmatrix is tuned to solve for a particular mode order such as monopoles, dipoles, ..., and through an examination of the eigenvectors, the dominant polarization and field localization were obtained. The computation process was highlighted for three different photonic structures. Steady 
states of a 12-fold quasi-crystal were determined for monopoles and dipoles and the field profiles plotted for highly localized central states as well as a less localized surface state. The rotational mode orders of 20 and 24 were obtained for a 3-D silicon ring resonator. The final application of the technique demonstrates that modes of photonic crystal fibers can be determined when the z-axis propagation constant is included in the eigenmatrix construction. The technique presented here is suitable for structures that present in-plane rotational symmetry about a central point and directly returns the steady states. Such a computation technique complements nicely the plane-wave method for determining band structures in translationally symmetric structures.

\section{Acknowledgements}

The authors thank NSERC for fundingthis and other research.

\section{Appendix A}

The nine equations of the matrix elements of (3) are:

$$
\begin{aligned}
& R_{r}=\mathrm{N} \sum_{r \Omega} \kappa^{\mathrm{r}} \kappa^{\Omega}\left(\left[q_{\mathrm{r}} q_{\Omega}+q_{r}^{2}\right] S_{-1}\left(p_{r}, p_{\Omega}, p_{r^{*}}\right)\right. \\
& \left.+\left[G_{n_{\mathrm{r}}} G_{n_{\Omega}}+G_{n_{\mathrm{r}}}{ }^{2}\right] R^{2} S_{1}\left(p_{r}, p_{\Omega}, p_{r^{*}}\right)\right) \\
& \varphi_{r}=\mathrm{N} \sum_{\varphi \Omega} \kappa^{\varphi} \kappa^{\Omega} j\left(q_{\Omega}+q_{\varphi}\right)\left[S_{-1}\left(p_{\varphi}, p_{\Omega}, p_{r^{*}}\right)-\left(\rho_{p_{\varphi}}\right) T_{o}\left(p_{\varphi}, p_{\Omega}, p_{r^{*}}\right)\right] \\
& Z_{r}=N \sum_{z \Omega} \kappa^{\mathrm{z}} \kappa^{\Omega}\left(-j \rho_{p_{\mathrm{z}}} R\right)\left(G_{n_{\Omega}}+G_{n_{\mathrm{z}}}\right) T_{1}\left(p_{z}, p_{\Omega}, p_{r^{*}}\right) \\
& R_{\varphi}=N \sum_{\mathrm{r} \Omega} \kappa^{\mathrm{r}} \kappa^{\Omega}\left[-j q_{r} S_{-1}\left(p_{r^{\prime}}, p_{\Omega}, p_{\varphi^{*}}\right)-j q_{r} \rho_{p_{r}} T_{0}\left(p_{r}, p_{\Omega}, p_{\varphi^{*}}\right)\right. \\
& \left.-j q_{r} \rho_{p_{\Omega}} U_{0}\left(p_{r}, p_{\Omega}, p_{\varphi^{*}}\right)\right] \\
& \varphi_{\varphi}=N \sum_{\varphi \Omega} \kappa^{\varphi} \kappa^{\Omega} b^{2}\left[\left\{G_{n_{\varphi}} G_{n_{\Omega}}+G_{n_{\varphi}}^{2}+\frac{\rho_{p_{\varphi}}^{2}}{b^{2}}\right\} S_{1}\left(p_{\varphi}, p_{\Omega}, p_{\varphi^{*}}\right)\right. \\
& +\frac{1}{b^{2}} S_{-1}\left(p_{\varphi}, p_{\Omega}, p_{\varphi^{*}}\right)+\left(\frac{\rho_{p_{\Omega}}}{b^{2}}\right) U_{0}\left(p_{\varphi}, p_{\Omega}, p_{\varphi^{*}}\right) \\
& \left.+\left(-\frac{\rho_{p_{\varphi}} \rho_{p_{\Omega}}}{b^{2}}\right) V_{1}\left(p_{\varphi}, p_{\Omega}, p_{\varphi^{*}}\right)\right] \\
& Z_{\varphi}=N \sum_{z \Omega} \kappa^{z} \kappa^{\Omega} b\left(-q_{z} G_{n_{\Omega}}-q_{z} G_{n_{z}}\right) S_{0}\left(p_{\varphi}, p_{\Omega}, p_{\varphi^{*}}\right) \\
& R_{z}=N \sum_{r \Omega} \kappa^{r} \kappa^{\Omega} R^{2}\left[\left(\frac{j G_{n_{r}}}{b}\right) S_{o}\left(p_{r}, p_{\Omega}, p_{z^{*}}\right)+\left(-\frac{j G_{n_{r}} \rho_{p_{r}}}{b}\right) T_{1}\left(p_{r}, p_{\Omega}, p_{z^{*}}\right)\right. \\
& \left.+\left(-\frac{j G_{n_{r}} \rho_{p_{\Omega}}}{b}\right) U_{1}\left(p_{r}, p_{\Omega}, p_{z^{*}}\right)\right] \\
& \varphi_{z}=N \sum_{\varphi \Omega} \kappa^{\varphi} \kappa^{\Omega} b\left(-G_{n_{\varphi}} q_{\Omega}-G_{n_{\varphi}} q_{\varphi}\right) S_{0}\left(p_{\varphi}, p_{\Omega}, p_{z^{*}}\right) \\
& Z_{z}=N \sum_{z \Omega} \kappa^{z} \kappa^{\Omega}\left[\rho_{p_{z}}^{2} S_{1}\left(p_{z}, p_{\Omega}, p_{z^{*}}\right)+\left(q_{z} q_{\Omega}+q_{z}{ }^{2}\right) S_{-1}\left(p_{z}, p_{\Omega}, p_{z^{*}}\right)\right. \\
& \left.-\left(\rho_{p_{z}} \rho_{p_{\Omega}}\right) V_{1}\left(p_{z}, p_{\Omega}, p_{z^{*}}\right)\right]
\end{aligned}
$$

Were the factor $N=\frac{\llbracket 2 \rrbracket}{\left[R J_{1}\left(\rho_{p_{\mathrm{r}^{*}}}\right)\right]^{2}}$ if $q_{i}+q_{\Omega}=q_{i^{*}}$ and $n_{i}+n_{\Omega}=n_{i^{*}}(i$ indicates a particular field component $)$ or zero otherwise. Within these expressions the $(S, T, U, V)$ are integrals involving the Bessel function obtained when applying the orthogonality condition and are:

$$
\begin{aligned}
S_{1}\left(p_{r}, p_{\Omega}, p_{r^{*}}\right) & =\int_{0}^{1} J_{o}\left(\rho_{p_{r}} \lambda^{\prime}\right) J_{o}\left(\rho_{p_{\Omega}} \lambda^{\prime}\right) J_{o}\left(\rho_{p_{\mathrm{r}}} \lambda^{\prime}\right) \lambda^{\prime} d \lambda^{\prime} \\
S_{o}\left(p_{r}, p_{\Omega}, p_{z^{*}}\right) & =\int_{0}^{1} J_{o}\left(\rho_{p_{r}} \lambda^{\prime}\right) J_{o}\left(\rho_{p_{\Omega}} \lambda^{\prime}\right) J_{o}\left(\rho_{p_{z^{*}}} \lambda^{\prime}\right) d \lambda^{\prime} \\
S_{-1}\left(p_{r}, p_{\Omega}, p_{r^{*}}\right) & =\int_{0}^{1} \frac{1}{\lambda^{\prime}} J_{o}\left(\rho_{p_{r}} \lambda^{\prime}\right) J_{o}\left(\rho_{p_{\Omega}} \lambda^{\prime}\right) J_{o}\left(\rho_{p_{\mathrm{r}}} \lambda^{\prime}\right) d \lambda^{\prime} \\
T_{1}\left(p_{z}, p_{\Omega}, p_{r^{*}}\right) & =\int_{0}^{1} J_{1}\left(\rho_{p_{z}} \lambda^{\prime}\right) J_{o}\left(\rho_{p_{\Omega}} \lambda^{\prime}\right) J_{o}\left(\rho_{p_{\mathrm{r}}}{ }^{*} \lambda^{\prime}\right) \lambda^{\prime} d \lambda^{\prime} \\
T_{o}\left(p_{\varphi}, p_{\Omega}, p_{r^{*}}\right) & =\int_{0}^{1} J_{1}\left(\rho_{p_{\varphi}} \lambda^{\prime}\right) J_{o}\left(\rho_{p_{\Omega}} \lambda^{\prime}\right) J_{o}\left(\rho_{p_{\mathrm{r}}}{ }^{*} \lambda^{\prime}\right) d \lambda^{\prime} \\
U_{1}\left(p_{r}, p_{\Omega}, p_{z^{*}}\right) & =\int_{0}^{1} J_{0}\left(\rho_{p_{r}} \lambda^{\prime}\right) J_{1}\left(\rho_{p_{\Omega}} \lambda^{\prime}\right) J_{o}\left(\rho_{p_{z^{*}}} \lambda^{\prime}\right) \lambda^{\prime} d \lambda^{\prime} \\
U_{0}\left(p_{r}, p_{\Omega}, p_{\varphi^{*}}\right) & =\int_{0}^{1} J_{0}\left(\rho_{p_{r}} \lambda^{\prime}\right) J_{1}\left(\rho_{p_{\Omega}} \lambda^{\prime}\right) J_{o}\left(\rho_{p_{\varphi^{*}}} \lambda^{\prime}\right) d \lambda^{\prime} \\
V_{1}\left(p_{z}, p_{\Omega}, p_{z^{*}}\right) & =\int_{0}^{1} J_{1}\left(\rho_{p_{z}} \lambda^{\prime}\right) J_{1}\left(\rho_{p_{\Omega}} \lambda^{\prime}\right) J_{o}\left(\rho_{p_{z^{*}}}{ }{ }^{\prime}\right) \lambda^{\prime} d \lambda^{\prime}
\end{aligned}
$$

Note: It is common to use $J_{m}$ instead of $J_{o}$ in the basis function expansion. The use of $J_{m}$ results in an unmanageablelarge set of integrals of the $(S, T, U, V)$ type, one set for each order $m$. The use of $J_{o}$ provides a finite set. These integrals are calculated once and used for all computations for any optical structure.

\section{References}

[1] S. Johnson and J. Joannopoulos, "Photonic crystals; The road from theory to practice," Kluwer Academic Publishers, Boston, 2002, pp. 20, 46.

[2] R. Gauthier and K. Mnaymneh, "Photonic band gap properties of 12-fold quasi-crystal determined through FDTD analysis," Opt. Express 13, 1985-1998 (2005).

[3] X. Qianfan, D. Fattal and R. G. Beausoleil, "Silicon microring resonators with $1.5-\mu \mathrm{m}$ radius," Optics Express, Vol. 16 Issue 6, pp.4309-4315 (2008).

[4] T. A. Birks , J. C. Knight, P. St. J. Russell, "Endlessly single-mode photonic crystal fiber," Opt. Lett. 22, pp. 961-963 (1997).

[5] "This is not a reference. It is the web address of the journal." http:/www.sciencepublishinggroup.com/journal/guideforaut hors.aspx?journalid=129 